\title{
The Maximum Limit of Rotational Energy Transfer in Molecular Collisions and the Hard Ellipsoid Potential Model
}

\author{
N.K. DABKARA \\ Department of Physics, Government Post Graduate College \\ Neemuch M.P., 458441, India
}

(Received April 20, 2001)

\begin{abstract}
The maximum limit of rotational energy transfer was investigated over a wide range of collision energy, reduced mass of the system, potential functions, and potential parameters for the $\mathrm{N}_{2}-\mathrm{Ne}$ system. The classical limit of maximum rotational energy transfer, $(\Delta E)_{\max }$, was reviewed for a hard ellipsoid potential model. It was found that $(\Delta E)_{\max }$ predicted by the hard ellipsoid model is comparable to a well-known parameter $|\Delta E|^{*}$ given by the power-gap law. The numerical equivalence of $|\Delta E|^{*}$ with $(\Delta E)_{\max }$ suggests that the value of $(\Delta E)_{\max }$ given by hard ellipsoid potential model can be used as one parameter $|\Delta E|^{*}$ of the power-gap law.
\end{abstract}

PACS numbers: $34.50 . \mathrm{Ez}, 34.50 .-\mathrm{s}$

\section{Introduction}

One of the important features regarding rotational energy transfer (RET) in atom-diatom collisions is to explore the region of molecular interaction where there is maximum exchange between the orbital angular momentum and the angular momentum of the molecule. It has been noted by several workers [1-6] in recent years that when an atom approaches a diatomic molecule the maximum amount of conversion of orbital angular momentum into the angular momentum of the molecule, or vice versa, occurs near the classical turning point.

Mc Caffery and co-workers [3-6] explored various aspects of RET by treating the conversion of orbital angular momentum to the angular momentum of the molecule at the repulsive wall of anisotropic intermolecular potential. They obtained the maximum classical limit of rotational energy transfer by using hard 
ellipsoid potential model. This model treats the target as a hard core ellipsoid and the collision between an atom and a diatom is considered equivalent to the collision between the atom and the hard core ellipsoid.

Considering the sum of the orbital angular momentum, $\boldsymbol{r} \times \boldsymbol{p}$, and the angular momentum of the molecule, $\boldsymbol{J}$, as a total angular momentum, $\boldsymbol{J}_{\mathrm{T}}$, the conservation of angular momentum can be expressed as

$$
\Delta \boldsymbol{J}_{\mathrm{T}}=\Delta(\boldsymbol{r} \times \boldsymbol{p})+\Delta \boldsymbol{J}=0,
$$

which gives

$$
\Delta \boldsymbol{J}=-r \times \Delta p,
$$

if $r$ is treated as constant.

The relationship between the shape of the potential surface and the energy transfer is crucial for understanding collision processes of atoms and molecules. The link between the two is provided by the general quantum theory of collisions for hard shapes. The problem of the rotationally inelastic collision of a particle with a hard ellipsoid potential [7-9] can be solved using the three principles of conservation: the total energy conservation, the linear momentum conservation, and the angular momentum conservation. Following Bosanac [8], Osborne and Mc Caffery [6] assumed the target as a hard core ellipsoid and computed the maximum possible value of $r \times \Delta p$ at the potential wall to arrive at the maximum limit of angular momentum transfer in terms of the dynamical parameters of the system. Recently, Agrawal and co-workers [10-12] have noted that the classical limit of the rotational energy transfer, $(\Delta E)_{\max }$, predicted by the hard ellipsoid model is comparable to a well-known parameter $|\Delta E|^{*}$ given by the power-gap law [13] and the RET cross-sections computed on the real potentials.

One more interesting point may be to study the above-mentioned classical limit of the angular momentum transfer in quantum mechanical cross-sections. Owing to quantum mechanical tunnelling and the softness of the realistic potentials, one expects the probability of a rotational transitions to be nonzero even in the classically forbidden region predicted by Bosanac [8] on the basis of the hard ellipsoid potential and Eq. (2). However, we shall see that the scaling and fitting laws [13-17] such as the power-gap law [15] will prove useful in separating the classically allowed and classically forbidden regions.

In this study, in addition to the validity of the hard ellipsoid potential model, we shall also reconfirm that the division between the classically allowed and forbidden transitions given by the power-gap law is excellent.

The classical limit of the angular momentum transfer for the hard ellipsoid potential model is reviewed in Sec. 2. The computational details and the procedure for determination of the classical limit of rotational energy transfer are then given in Sec. 3. The numerical results are presented and discussed in Sec. 4. Finally, the conclusions are summarized in Sec. 5. 


\section{Hard ellipsoid potential model}

We present here a simplified model for the maximum limit of angular momentum transfer. The hard ellipsoid potential model is shown in Fig. 1. The point $O$ in the figure refers to the centre of mass of the diatomic molecule. Before the collision it is assumed that the ellipsoid is non-rotating. At the point of impact, $M$, the component of linear momentum tangential to the surface of ellipsoid does not change its value. Therefore, the direction of change in momentum, $\Delta p$, due to collision with the hard ellipsoid potential would be along the normal $I M$ in the figure. To determine the rotational angular momentum $\boldsymbol{J}$ of the molecule in the final state we use the principle of conservation of angular momentum. After the collision for such a change in the linear momentum the magnitude of the conversion of orbital angular momentum of the system into the angular momentum of the diatomic molecule given by Eq. (2) would be

$$
\Delta \boldsymbol{J}=b_{n}|\Delta \boldsymbol{p}|,
$$

where $b_{n}$ is the length of the perpendicular $O P$ on the normal $I M N$. By using the properties of an ellipsoid one can write the following equation for the maximum possible value of $b_{n}$ :

$$
\left(b_{n}\right)_{\max }=A-B,
$$

where $A$ and $B$ are the lengths of the semi-major and semi-minor axes, respectively.

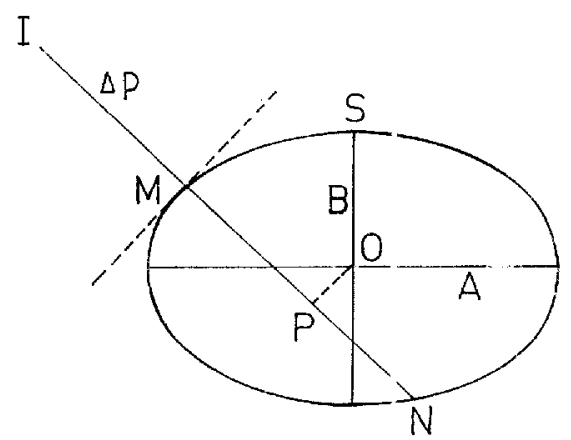

Fig. 1. A hard ellipsoid model of the rotationally inelastic atom-diatom collision.

Further, for the maximum possible value of $\Delta p$ we can write

$$
|\Delta p|_{\max }=p-\left(-p^{\prime}\right)=\sqrt{2 \mu E}-\left(-\sqrt{2 \mu E^{\prime}}\right)=\sqrt{2 \mu}\left(\sqrt{E}+\sqrt{E^{\prime}}\right),
$$

where $\mu$ is the reduced mass of the colliding system, and $E$ and $E^{\prime}$ are the initial and final translational kinetic energies of the system, respectively.

Combining Eqs. (3)-(5), we can now write the following relation for the classical limit of the angular momentum transfer:

$$
(\Delta J)_{\max }=\sqrt{2 \mu}\left(\sqrt{E}+\sqrt{E^{\prime}}\right)(A-B) .
$$


From the above expression the limit of the rotational energy transfer in the molecule can be easily obtained. For simplicity, if the diatomic molecule is considered initially in the ground state, then the expression for the maximum amount of rotational energy transfer would be

$$
(\Delta E)_{\max }=\left[(\Delta J)_{\max }\right]^{2} / 2 I=(\mu / I)(A-B)^{2}\left(E+E^{\prime}+2 \sqrt{E E^{\prime}}\right),
$$

where $I$ is the moment of inertia of the diatomic molecule. Equation (7) together with the following energy conservation equation:

$$
E^{\prime}=E-(\Delta E)_{\max }
$$

can be used to compute $(\Delta E)_{\max }$ from knowledge of $E, A, B, \mu$, and $I$.

\section{Computation of $|\Delta E|^{*}$}

The parameter $|\Delta E|^{*}$ is determined with the use of integral inelastic cross-sections (IICS) obtained from scattering calculations and the power-gap law.

\subsection{Potential energy surfaces}

For the computation of IICS the homonuclear diatomic molecule, $\mathrm{N}_{2}$, is treated as a rigid rotor and the interaction between the molecule and the atom, $\mathrm{Ne}$, is taken as a pairwise sum of the potential terms, i.e.

$$
V=V\left(r_{1}\right)+V\left(r_{2}\right)
$$

where $r_{1}$ and $r_{2}$ are the $\mathrm{N}^{(1)}-\mathrm{Ne}$ and $\mathrm{N}^{(2)}-\mathrm{Ne}$ distances, respectively, as shown in Fig. 2.

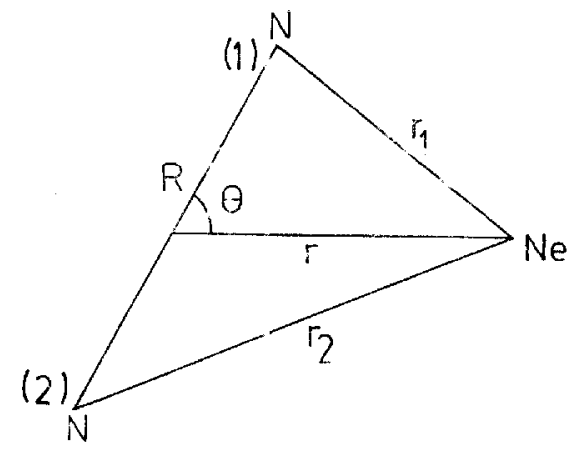

Fig. 2. Coordinates for the rigid rotor $\mathrm{N}_{2}-\mathrm{Ne}$ system.

For $V\left(r_{i}\right)$ the following general form of the Lennard-Jones ( $\left.\mathrm{L}-\mathrm{J}\right)$ potential is taken with different values of $n$ and $m$ :

$$
V\left(r_{i}\right)=\varepsilon\left\{[m /(n-m)]\left(r_{0} / r_{i}\right)^{n}-[n /(n-m)]\left(r_{0} / r_{i}\right)^{m}\right\}, \quad i=1,2, \quad(10)
$$

where $r_{0}$ and $\varepsilon$ are taken $[18,19]$ as $3.63 \AA$ and $5.352 \mathrm{meV}$, respectively. 
The variation in the potential function is obtained by putting $n=9$ and 12 in Eq. (10), keeping $m=6$, giving the potential functions which we shall denote as $V(9,6)$ and $V(12,6)$.

In addition to the above-mentioned potential functions, purely repulsive terms of the potential functions have also been investigated. It is convenient to denote such potentials by the notation $V_{\mathrm{R}}(n)$, which has been obtained by deleting the attractive term from the potential $V(n, m)$.

\subsection{Computation of cross-sections}

The integral inelastic cross-sections have been computed using the modified infinite order sudden approximation (IOSAM) [20]. Depending on the energy and the mass of the system, $\mathrm{X}_{2}-\mathrm{Ne}$, the number of phase shifts were varied in the range 100 to 400 . The phase shifts have been computed using a 10-point Guass-Mehler quadrature of the WKB phase shift equation as described by Pack [21].

\subsection{The power-gap law and the classical limit of RET}

According to the power-gap law [15] the cross-sections, $\sigma\left(j_{\mathrm{i}} \rightarrow j_{\mathrm{f}}\right)$, can be expressed as

$$
\sigma\left(j_{\mathrm{i}} \rightarrow j_{\mathrm{f}}\right)=a\left(2 j_{\mathrm{f}}+1\right)\left(T_{\mathrm{f}} / T_{\mathrm{i}}\right)^{1 / 2}|\Delta E|^{-\gamma},
$$

where $j_{\mathrm{i}}$ and $j_{\mathrm{f}}$ are the initial and final rotational quantum numbers, $a$ and $\gamma$ are the fitting parameters, $T_{\mathrm{f}}$ and $T_{\mathrm{i}}$ are final and initial translational energies and $|\Delta E|$ is the energy gap between initial and final rotational levels.

Equation (11) gives the following equation which can be used to separate the two regions:

$$
Y=-\gamma X+\ln a,
$$

where

$$
Y=\ln \left[\sigma\left(j_{\mathrm{i}} \rightarrow j_{\mathrm{f}}\right)\left(T_{\mathrm{i}} / T_{\mathrm{f}}\right)^{1 / 2} /\left(2 j_{\mathrm{f}}+1\right)\right]
$$

and

$$
X=\ln |\Delta E|
$$

A typical $X-Y$ plot which shows the existence of two straight lines signifying the two regions is given in Fig. 3. The location of the critical point has been indicated by the arrow in the figure. For all sets of computed cross-sections, $|\Delta E|^{*}$ has been obtained by such plots. The values of $|\Delta E|^{*}$ are compared with the corresponding values of $(\Delta E)_{\max }$ given by Eq. (7). 


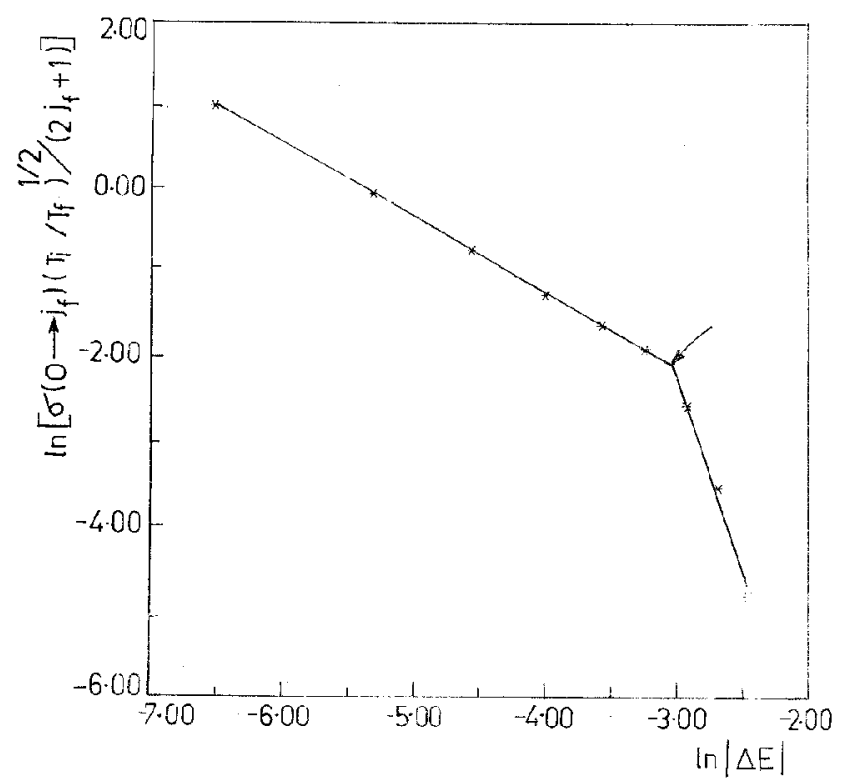

Fig. 3. $\ln \left[\sigma\left(j_{\mathrm{i}} \rightarrow j_{\mathrm{f}}\right)\left(T_{\mathrm{i}} / T_{\mathrm{f}}\right)^{1 / 2} /\left(2 j_{\mathrm{f}}+1\right)\right]$ versus $\ln |\Delta E|$ for $j_{\mathrm{i}}=0$ at $E=0.1 \mathrm{eV}$ for $V_{\mathrm{R}}(9)$ potential. $|\Delta E|^{*}$ point is shown by an arrow. The units of $\Delta E$ and $\sigma$ are $\mathrm{eV}$ and $\AA^{2}$, respectively.

\section{Results and discussion}

\subsection{Effect of energy}

The effect of varying energy on slopes, $|\Delta E|^{*}$ and $\left(j_{\mathrm{f}}\right)^{*}$ given by the scattering calculations for the potential $V(9,6)$ and $V_{\mathbf{R}}(9)$ is shown in Table $\mathbf{I}$. In all the calculations the molecule, $\mathrm{N}_{2}$, was taken initially in the rotational ground state. For comparison the values of $(\Delta E)$ max predicted by the hard ellipsoid model are also listed in the Table. The listed values of $(\Delta E)_{\max }$, in general, do not correspond to definite quantum levels. To translate the classical information contained in $(\Delta E)_{\max }$ into the quantum picture, the permissible (even) value of rotational quantum number, $\left(j_{\mathrm{f}}\right)_{\max }$, corresponding to the rotational energy level just before the occurrence of classical rotational energy $(\Delta E)_{\max }$ is also listed in the Table.

A comparison of $(\Delta E)_{\max }$ given by the hard ellipsoid model with the corresponding data obtained by the scattering method shows that they are in good agreement. However, in terms of the quantum level expressed by $\left(j_{\mathrm{f}}\right)_{\max }$ one notes that the difference between the scattering results and that of ellipsoid model extends only up to the next permissible level.

Another important parameter is $\gamma_{\text {low }}$. For a given potential we see that $\gamma_{\text {low }}$ is insensitive to the change in the collision energy. The data reported in Table I also reveal that $\gamma_{\text {low }}$ increases with the removal of attractive term in the potential. 
TABLE I

Comparison of maximum amount of rotational energy transfer $(\Delta E)_{\max }$ and $\left(j_{\mathrm{f}}\right)_{\max }$ values given by the hard ellipsoid potential model and those obtained by using the scattering results for $\mathrm{N}_{2}-\mathrm{Ne}$ system.

\begin{tabular}{c|c|c|c|c|c|c|c}
\hline \hline Potential & $\begin{array}{c}\text { Energy } \\
{[\mathrm{eV}]}\end{array}$ & $\gamma_{\text {low }}{ }^{a}$ & $\gamma_{\text {high }}{ }^{a}$ & \multicolumn{2}{|c|}{$(\Delta E)_{\max }[\mathrm{eV}]$} & \multicolumn{2}{|c}{$\left(j_{\mathrm{f}}\right)_{\max }$} \\
\cline { 5 - 8 } & & & $\begin{array}{c}\text { Scat- } \\
\text { tering }\end{array}$ & $\begin{array}{c}\text { Ellipsoid } \\
\text { model }\end{array}$ & $\begin{array}{c}\text { Scat- } \\
\text { tering }\end{array}$ & $\begin{array}{c}\text { Ellipsoid } \\
\text { model }\end{array}$ \\
\hline$V(9,6)$ & 0.10 & 0.76 & 1.78 & 0.067 & 0.070 & 14 & 16 \\
& 0.15 & 0.78 & 3.47 & 0.116 & 0.104 & 20 & 18 \\
& 0.20 & 0.79 & 2.21 & 0.141 & 0.139 & 22 & 22 \\
& 0.25 & 0.80 & 2.97 & 0.189 & 0.175 & 26 & 24 \\
& 0.30 & 0.81 & 4.08 & 0.243 & 0.210 & 30 & 28 \\
& 0.35 & 0.81 & 2.49 & 0.254 & 0.246 & 30 & 30 \\
\hline$V_{\mathrm{R}}(9)$ & 0.10 & 0.90 & 4.54 & 0.048 & 0.057 & 12 & 14 \\
& 0.15 & 0.94 & 6.74 & 0.091 & 0.089 & 18 & 18 \\
& 0.20 & 0.92 & 5.59 & 0.116 & 0.121 & 20 & 20 \\
& 0.25 & 0.93 & 6.64 & 0.158 & 0.154 & 24 & 24 \\
& 0.30 & 0.92 & 6.90 & 0.193 & 0.187 & 26 & 26 \\
& 0.35 & 0.92 & 6.39 & 0.225 & 0.220 & 28 & 28 \\
& 0.40 & 0.93 & 7.05 & 0.273 & 0.254 & 32 & 30 \\
\hline
\end{tabular}

${ }^{a}$ The units of $\gamma_{\text {low }}$ and $\gamma_{\text {high }}$ are such that in Eq. (11), cross-section is in $\AA^{2}$ and $\Delta E$ is in $\mathrm{eV}$.

The values of $\gamma_{\text {high }}$, however, show a different trend. The energy dependence of these parameters is a matter of further studies.

\section{D. Effect of mass}

Table II gives the results for $\mathrm{X}_{2}-\mathrm{Ne}$ system having $r_{0}$ and $\varepsilon$ values of $\mathrm{N}_{2}-\mathrm{Ne}$ system and the masses of $\mathrm{X}_{2}$ are $24.0,28.0,32.0 \mathrm{amu}$ for the potential $V_{\mathrm{R}}(12)$ at different collision energies. A very good agreement between the $(\Delta E)_{\max }$ values given by the hard ellipsoid model and the scattering calculations is seen from Table II. Further, a decrease in $(\Delta E)_{\max }$ values with the increase in mass of the diatom, $\mathrm{X}_{2}$, is also seen from the Table.

For a system to exhibit angular momentum constraint, the final rotational state of the molecule must be energetically accessible to eliminate any energetic restrictions. Thus the $(\Delta E)_{\max }$ values must be smaller than the available translational energy $E$. This gives

$$
(\Delta E)_{\max } / E<1
$$

or

$$
(\mu / I)(A-B)^{2}<1 \quad(\text { using Eq. (7)) }
$$


TABLE II

Comparison of maximum amount of rotational energy transfer $(\Delta E)_{\max }$ given by the hard ellipsoid potential model and those obtained by using the scattering results for $\mathrm{X}_{2}-\mathrm{Ne}$ system, as a function of mass of the diatom, $\mathrm{X}_{2}$, at different collision energies. Potential parameters of $\mathrm{X}_{2}$ are taken as of $\mathrm{N}_{2}$. Potential $V_{\mathrm{R}}(12)$ is used.

\begin{tabular}{c|c|c|c|c|c|c}
\hline \hline \multirow{2}{*}{$\begin{array}{c}\text { Energy } \\
\text { in eV }\end{array}$} & \multicolumn{5}{|c}{ Mass of $\mathrm{X}_{2}$ in amu } \\
\cline { 2 - 7 } & \multicolumn{2}{|c}{24.0} & \multicolumn{5}{|c}{28.0} & \multicolumn{3}{c}{$(\Delta E)_{\max }[\mathrm{eV}]$} \\
\hline & $\begin{array}{c}\text { Scat- } \\
\text { tering }\end{array}$ & $\begin{array}{c}\text { Ellipsoid } \\
\text { model }\end{array}$ & $\begin{array}{c}\text { Scat- } \\
\text { tering }\end{array}$ & $\begin{array}{c}\text { Ellipsoid } \\
\text { model }\end{array}$ & $\begin{array}{c}\text { Scat- } \\
\text { tering }\end{array}$ & $\begin{array}{c}\text { Ellipsoid } \\
\text { model }\end{array}$ \\
\hline 0.10 & 0.068 & 0.069 & 0.060 & 0.066 & 0.056 & 0.063 \\
0.15 & 0.109 & 0.106 & 0.099 & 0.101 & 0.097 & 0.096 \\
0.20 & 0.139 & 0.142 & 0.137 & 0.136 & 0.127 & 0.130 \\
0.25 & 0.186 & 0.180 & 0.184 & 0.171 & 0.169 & 0.164 \\
0.30 & 0.225 & 0.217 & 0.223 & 0.207 & 0.216 & 0.198 \\
0.35 & 0.275 & 0.255 & 0.257 & 0.243 & 0.249 & 0.232 \\
0.40 & 0.326 & 0.292 & 0.309 & 0.279 & 0.282 & 0.267
\end{tabular}

or

$$
\left(\mu / \mu_{m}\right)\left[(A-B) / R_{e}\right]^{2}<1 .
$$

where $\mu_{m}$ and $R_{e}$ are the reduced mass and bond length of the molecule. Thus the ratio $\mu / \mu_{m}$ is an important factor in determining whether a collision system is momentum or energy constrained. In $\mathrm{N}_{2}-\mathrm{Ne}$ system the order of $A-B$ is 0.47 and the bond length of $\mathrm{N}_{2}$ is $R_{\mathrm{e}}=1.094 \AA$. Hence $\left[(A-B) / R_{\mathrm{e}}\right]^{2}$ is $\approx 0.2$. Therefore the ratio $\mu / \mu_{m}$ should be smaller than 5 for the inequality in Eq. (15) to hold. In other cases all the transitions permitted by the energy conservation constraint would be possible.

\subsection{Effect of potential parameters $r_{0}$ and $\varepsilon$}

The parameters $A$ and $B$ do not appreciably change with energy $E$. For example, for $V_{\mathrm{R}}(9)$ potential $A$ decreases from 3.393 to 2.9826 and $A-B$ increases from 0.3862 to 0.4200 as $E$ increases from 0.1 to $0.4 \mathrm{eV}$, respectively.

Therefore, to study the dependence of $(\Delta E)_{\max }$ on $A-B$ as given by Eq. (7) we have varied the potential parameter $r_{0}$ from $0.5 R_{0}$ to $2.0 R_{0}$, where $R_{0}=$ $3.232 \AA$. The so obtained results for $\mathrm{N}_{2}-\mathrm{Ne}$ system at $E=0.1 \mathrm{eV}$ for $V_{\mathrm{R}}(9)$ potential are given in Table III. With such a change in the range parameters now $A$ varies from 1.9628 to $6.3274 \AA$ and $A-B$ ranges from 0.536 to 0.2396 as $r_{0}$ varies from $0.5 R_{0}$ to $2.0 R_{0}$, respectively, for $V_{\mathrm{R}}(9)$ potential. Thus the data presented in Table III exclusively demonstrate the effect of variation of $A-B$ on $(\Delta E)_{\max }$. 
TABLE III

Comparison of maximum amount of rotational energy transfer $(\Delta E)_{\max }$ and $\left(j_{\mathrm{f}}\right)_{\max }$ values given by the hard ellipsoid potential model and those obtained by using the scattering results for $\mathrm{N}_{2}-\mathrm{Ne}$ system as a function of potential parameter, $r_{0}$, at collision energy $E=0.1 \mathrm{eV}\left(R_{0}=3.232 \AA\right)$. Potential $V_{\mathrm{R}}(9)$ is used.

\begin{tabular}{c|c|c|c|c|c|c}
\hline \hline$r_{0} / R_{0}$ & $A$ & $B$ & \multicolumn{2}{|c|}{$(\Delta E)_{\max }[\mathrm{eV}]$} & \multicolumn{2}{|c}{$\left(j_{\mathrm{f}}\right)_{\max }$} \\
\cline { 4 - 7 } & {$[\AA]$} & {$[\AA]$} & Scattering & $\begin{array}{c}\text { Ellipsoid } \\
\text { model }\end{array}$ & Scattering & $\begin{array}{c}\text { Ellipsoid } \\
\text { model }\end{array}$ \\
\hline 0.5 & 1.9628 & 1.4268 & 0.076 & 0.082 & 16 & 16 \\
0.7 & 2.5320 & 2.0682 & 0.063 & 0.071 & 14 & 16 \\
1.0 & 3.3930 & 3.0068 & 0.048 & 0.057 & 12 & 14 \\
1.3 & 4.2640 & 3.9352 & 0.043 & 0.046 & 12 & 12 \\
1.5 & 4.8496 & 4.5514 & 0.034 & 0.039 & 10 & 12 \\
2.0 & 6.3274 & 6.0878 & 0.025 & 0.028 & 8 & 8 \\
\end{tabular}

TABLE IV

Comparison of maximum amount of rotational energy transfer $(\Delta E)_{\max }$ and $\left(j_{\mathrm{f}}\right)_{\max }$ values given by the hard ellipsoid potential model and those obtained by using the scattering results for $\mathrm{N}_{2}-\mathrm{Ne}$ system as a function of potential parameter, $\varepsilon$, at collision energy $E=0.1 \mathrm{eV}\left(\varepsilon_{0}=5.351947 \mathrm{meV}\right)$. Potential $V_{\mathrm{R}}(12)$ is used.

\begin{tabular}{c|c|c|c|c|c|c}
\hline \hline$\varepsilon / \varepsilon_{0}$ & $A$ & $B$ & \multicolumn{2}{|c|}{$(\Delta E)_{\max }[\mathrm{eV}]$} & \multicolumn{2}{|c}{$\left(j_{\mathrm{f}}\right)_{\max }$} \\
\cline { 4 - 7 } & {$[\AA]$} & {$[\AA]$} & Scattering & $\begin{array}{c}\text { Ellipsoid } \\
\text { model }\end{array}$ & Scattering & $\begin{array}{c}\text { Ellipsoid } \\
\text { model }\end{array}$ \\
\hline 0.5 & 3.2332 & 2.7888 & 0.062 & 0.068 & 14 & 14 \\
1.0 & 3.3938 & 2.9610 & 0.060 & 0.066 & 14 & 14 \\
1.5 & 3.4942 & 3.0660 & 0.060 & 0.065 & 14 & 14 \\
2.0 & 3.5640 & 3.1428 & 0.059 & 0.064 & 14 & 14 \\
5.0 & 3.8052 & 3.3994 & 0.057 & 0.061 & 14 & 14 \\
10.0 & 4.0006 & 3.6066 & 0.049 & 0.059 & 12 & 14 \\
20.0 & 4.2078 & 3.8260 & 0.048 & 0.056 & 12 & 14
\end{tabular}

Here again we see a very good agreement between the scattering results and those given by Eq. ( 7$)$ for $(\Delta E)_{\max }$ and $\left(j_{\mathrm{f}}\right)_{\max }$.

Unlike the effect of $r_{0}$, the parameter $\varepsilon$ has small effect on $A-B$. Such an effect of varying $\varepsilon$ from $0.5 \varepsilon_{0}$ to $20.0 \varepsilon_{0}$, where $\varepsilon_{0}=5.352 \mathrm{meV}$, is shown in Table IV at $E=0.1 \mathrm{eV}$. Here $A-B$ decreases by about $14 \%$ as $\varepsilon$ increases from $0.5 \varepsilon_{0}$ to $20.0 \varepsilon_{0}$ for $V_{\mathrm{R}}(12)$ potential. The data presented in Table IV also show a very good agreement between the scattering results and those given by the hard ellipsoid model. 


\section{Summary}

The maximum limit of rotational energy transfer in collisions of $\mathrm{N}_{2}$ with $\mathrm{Ne}$ was investigated over a wide range of energies, reduced mass of the system, potential functions, and potential parameters. Further, the classical limit of maximum rotational energy transfer was reviewed for a hard ellipsoid potential model. Corresponding to the classical limit $(\Delta E)_{\max }$, the final rotational quantum number $\left(j_{\mathrm{f}}\right)_{\max }$ was also computed in each case, considering the molecule initially in the ground state. A good agreement between the $|\Delta E|^{*}$ so obtained by power-gap law and $(\Delta E)_{\max }$ given by Eq. (7) clearly signify that the region $|\Delta E|>|\Delta E|^{*}$ is due to the departure of the real potential from the hard ellipsoid potential and quantum mechanical tunnelling.

Also the systematic decrease in $(\Delta E)_{\max }$ with the increase in the reduced mass of the system was verified for $\mathrm{X}_{2}-\mathrm{Ne}$ system, where diatom $\mathrm{X}_{2}$ has all properties of $\mathrm{N}_{2}$ except the mass, which is taken as $24.0,28.0,32.0 \mathrm{amu}$. The $|\Delta E|^{*}$ values given by the scattering results are found to be in good agreement with the $(\Delta E)_{\max }$ values obtained using the hard ellipsoid potential model.

The dependence of $(\Delta E)_{\max }$ and $\left(j_{\mathrm{f}}\right)_{\max }$ for $\mathrm{N}_{2}-\mathrm{Ne}$ system was investigated for different types of potential surfaces over a wide range of initial collision energy and potential parameters. In all cases it is found that the agreement between $(\Delta E)_{\max }$ and $|\Delta E|^{*}$ is good.

The equivalence of $|\Delta E|^{*}$ and $(\Delta E)_{\max }$ suggests that the value of $(\Delta E)_{\max }$ given by Eq. (7) can be used as one parameter $|\Delta E|^{*}$ of the power-gap law. The physical explanation of the other four parameters $a_{\text {low }}, a_{\text {high }}, \gamma_{\text {low }}$, and $\gamma_{\text {high }}$ of the power-gap law is, however, a matter of further research.

\section{Acknowledgment}

Author thanks to P.M. Agrawal for valuable suggestions and affectionate encouragement. Author also wishes to thank Ms. Anita Dabkara and Avijit for the active cooperation and inspiration during the course of this work. The support of the University Grants Commission, Central Regional Office, Bhopal, under grant No. F-4-158 (8)/99 (MRP/CRO) is gratefully acknowledged.

\section{References}

[1] S.D. Bosanac, N. Petrovic, Phys. Rev. A 41, 5909 (1990).

[2] F.A. Gianturco, J.P. Toennies, M. Bernardi, J. Chem. Soc. Faraday Trans, 87, 31 (1991).

[3] A.J. Mc Caffery, Z.T. Alwahabi, M.A. Osborne, C.J. Williams, J. Chem. Phys. 98, 4586 (1993).

[4] M.A. Osborne, A.J. Mc Caffery, J. Chem. Phys. 101, 5604 (1994). 
[5] M.A. Osborne, A.J. Marks, A.J. Mc Caffery, J. Phys. Chem. 100, 3888 (1996).

[6] M.A. Osborne, A.J. Mc Caffery, J. Phys. Chem. 100, 13346 (1996).

[7] D. Beck, U. Ross, W. Schepper, Z. Phys. A 293, 107 (1979).

[8] S. Bosanac, Phys. Rev. A 22, 2617 (1980).

[9] J.A. Serri, R.M. Bilotta, D.E. Pritchard, J. Chem. Phys. 77, 2940 (1982).

[10] P.M. Agrawal, S. Tilwanker, N.K. Dabkara, J. Chem. Phys. 108, 4854 (1998).

[11] P.M. Agrawal, S. Tilwanker, Acta Phys. Pol. A 93, 451 (1998).

[12] N.K. Dabkara, P.M. Agrawal, Chem. Phys. Lett. 299, 125 (1999).

[13] J.M. Huston, Ann. Rev. Phys. Chem. 41, 123 (1990).

[14] J.C. Polanyi, K.B. Woodall, J. Chem. Phys. 56, 1563 (1972).

[15] T.A. Brunner, N. Smith, A.W. Karp, D.E. Pritchard, J. Chem. Phys. 74, 3324 (1981).

[16] I. Noor Batcha, N. Sathyamurthy, Chem. Phys. Lett. 79, 264 (1981).

[17] J.I. Steinfeld, P. Ruttenberg, G. Millot, G. Fanjoux, B. Lovored, J. Phys. Chem. 98, 9638 (1991).

[18] American Institute of Physics Handbook, Ed. D.E. Gray, McGraw-Hill, New York 1957, p. 4.129 .

[19] R.A. Svehla, NASA Tech. Rep. TR R-132, 1962.

[20] P.M. Agrawal, L.M. Raff, J. Chem. Phys. 74, 3292 (1981).

[21] R.T. Pack, J. Chem. Phys. 60, 633 (1974). 